\title{
Representaciones maternas sobre salud y lactancia del hijo prematuro en hospitales con y sin certificación de Hospital Amigo del Niño y de la Madre
}

Josefina Gallegos-Martínez, ${ }^{1}$ Jaime Reyes-Hernández ${ }^{2}$

\begin{abstract}
Resumen
ANTECEDENTES: la prevalencia de la lactancia materna en prematuros mexicanos es baja, a pesar de ser una medida accesible y de bajo costo recomendada por la Organización Mundial de la Salud. Uno de los factores de abandono es la percepción de baja producción láctea.
\end{abstract}

OBJETIVO: comparar las representaciones maternas de salud y lactancia en hijos prematuros internados en hospitales de referencia, en tanto certificado o no como Hospital Amigo del Niño y de la Madre.

MATERIAL Y MÉTODOS: estudio cualitativo efectuado en dos hospitales de referencia: uno de ellos certificado como amigo del niño y la madre (17 madres y 1 padre de prematuros) y otro no certificado (13 madres). Instrumento de recolección: guía de entrevista semiestructurada que se realizó cara a cara por personal capacitado. Se aplicó análisis de contenido temático a las comunicaciones hasta aprehender las representaciones de acuerdo con el objetivo. Se obtuvo la aprobación de los comités de ética de cada hospital y los participantes firmaron el consentimiento informado.

RESULTADOS: se aprehendieron tres representaciones: 1) la saludcorporalidad del hijo prematuro internado; 2 ) elegir el mejor alimento la leche materna versus fórmula para el hijo prematuro y 3) las informaciones recibidas sobre lactancia materna.

CONCLUSIONES: la salud del hijo prematuro se representa en la corporalidad, principalmente el peso. En las representaciones maternas se manifestó la construcción de conocimiento de la leche como alimento "bueno", que les permitió optar, inicialmente, por alimentar al hijo con su leche. La percepción de producción insuficiente, la creencia de que la leche materna daña, la actividad laboral materna y la prescripción de sucedáneos posibilita el abandono de la lactancia materna. En el Hospital Amigo del Niño y de la Madre se denotó sistematización en pro de la lactancia, con personal e infraestructura asignados a esa labor; en el hospital no certificado se denotaron solamente acciones informativas verbales o gráficas.

PALABRAS CLAVE: lactancia materna, prematuro, representaciones.
${ }^{1}$ Doctora en Enfermería en salud pública y materno infantil. Profesora investigadora de tiempo completo en la Facultad de Enfermería y Nutrición. Miembro del Sistema Nacional de Investigadores nivel 1.

${ }^{2}$ Profesor-Investigador de Tiempo Completo. Líder del Cuerpo Académico Alimentos Nutrición y Salud. Facultad de Enfermería y Nutrición.

Universidad Autónoma de San Luis Potosí.

Recibido: 1 de enero 2017

Aceptado: 24 de julio 2017

Correspondencia

Jaime Reyes-Hernández

reyes.jaimeh@fcq.uaslp.mx

Este artículo debe citarse como

Gallegos-Martínez J, Reyes-Hernández J. Representaciones maternas sobre salud y lactancia del hijo prematuro en hospitales con y sin certificación de Hospital Amigo del Niño y de la Madre. Acta Pediatr Mex. 2018;39(2):99-108. 


\section{Maternal representations about health and breastfeeding of the premature child in hospitals with and without baby and mother friendly hospital initiative certification.}

\author{
Josefina Gallegos-Martínez, ${ }^{1}$ Jaime Reyes-Hernández ${ }^{2}$
}

\begin{abstract}
BACKGROUND: Breastfeeding in preterm infants in Mexico has a low prevalence despite being an affordable low-cost strategy recommended by WHO. One of the factors of abandonment is the maternal perception of low milk production.
\end{abstract}

OBJECTIVE: Comparing the maternal representations on the health of the premature child and breast-feeding, in two hospitals, one of them with Baby and Mother Friendly Hospital Initiative certification.

MATERIAL AND METHODS: A qualitative study was carried out in two reference hospitals, one of them certified as a friend of the child and the mother (17 mothers and 1 father of premature babies) and one without a certificate (13 mothers). Collection instrument: Semistructured interview guide, interviews were conducted face-to-face by trained personnel. Thematic contents analysis was applied to the communications to apprehend the maternal representations according to the objective. Approval was obtained from the ethics committees of each hospital and informed consent was signed by the participants.

RESULTS: Three representations were aprehended: 1 . The health related corporeality of the premature child hospitalized; 2. Selecting the best food, breast milk versus formula for the premature child; and 3. The information received on breastfeeding.

CONCLUSIONS: The health of the premature child is represented in the corporeality, mainly the weight. Breast milk was represented as "good" food, which helped to initially choose to breastfeed her child. The perception of insufficient production, belief about maternal milk that damages, maternal labor activity and the prescription of substitutes give the possibility of abandonment of breastfeeding. The certified Baby and Mother Friendly Hospital denotes systematization for breastfeeding, with personnel and infrastructure assigned to that work and in the non-certified hospital only verbal or graphic informative actions are denoted.

KEYWORDS: breastfeeding; preterm infants; representations

\footnotetext{
${ }^{1}$ Dra. en Enfermería en salud pública y materno infantil. Profesora investigadora de tiempo completo en la Facultad de Enfermería y Nutrición. Miembro del Sistema Nacional de Investigadores nivel 1.

${ }^{2}$ Profesor-Investigador de Tiempo Completo. Líder del Cuerpo Académico Alimentos Nutrición y Salud. Facultad de Enfermería y Nutrición.
}

Universidad Autónoma de San Luis Potosí.

Correspondence

Jaime Reyes-Hernández

reyes.jaimeh@fcq.uaslp.mx 


\section{INTRODUCCIÓN}

Uno de los objetivos del milenio, para cumplirse en 2025, es la reducción de la mortalidad neonatal en $50 \%$ en países con mortalidad $\geq 5$ por 1000 recién nacidos vivos registrados. La lactancia materna exclusiva, al menos por seis meses y extenderla con alimentación complementaria por dos años, es una estrategia de bajo costo, basada en evidencia, propuesta por la Organización Mundial de la Salud (OMS). ${ }^{1}$

El costo de la práctica inadecuada de la lactancia materna en México, de acuerdo con un estudio que tomó en cuenta a los sucedáneos de la leche materna y la incidencia y gastos por las enfermedades más frecuentes atribuibles a esa práctica inadecuada (diarrea aguda, infecciones respiratorias agudas y enterocolitis necrosante), se reportó en un rango de 745.6 a 2616.5 miIlones de dólares. ${ }^{2}$ En México se reportó, en la Encuesta Nacional de Salud y Nutrición 2012, que entre las diez primeras razones para no amamantar a niños menores de 24 meses por parte de madres entre 12 y 49 años es la prematurez. ${ }^{3}$ Del mismo modo, en un estudio de cohorte multicéntrico realizado en Australia, donde la lactancia exclusiva tiene una prevalencia mayor de $90 \%$ al egreso, la prematurez y bajo peso se reportaron como causas de cesación temprana de la lactancia materna exclusiva o mixta. ${ }^{4}$

En estudios al respecto se han reportado prevalencias variables de lactancia materna en prematuros: en Dinamarca, en donde en las unidades neonatales existe el contacto piel a piel, soporte para expresión láctea, alojamiento conjunto y presencia de los padres, en el National Cohort Study with High Breastfeeding Initiation Rates los resultados mostraron que 99\% de los prematuros tuvieron lactancia materna en el internamiento y al egreso $68 \%$ estaba en lactancia exclusiva. ${ }^{5}$
En Suecia, la norma cultural es pro-lactancia materna y amamantar forma parte importante de la buena maternidad, aún así, en las experiencias de madres suecas con hijos prematuros, para realizar la lactancia hubieron tres barreras reconocidas por ellas: el autoritarismo del personal, la falta de alivio a su sufrimiento y las experiencias dolorosas en la unidad neonatal. ${ }^{6}$ En el estudio sueco Swedish Nenatal Quality Register de 2004 se mostró una disminución global de la lactancia exclusiva en los prematuros de $59 \%$ y de $45 \%$ en $2013 .{ }^{7}$ En un estudio retrospectivo francés más de $66 \%$ de las madres deseó amamantar, pero al egreso solo lo hicieron $38 \%{ }^{8}$

En México, en un hospital pediátrico del noroeste del país, se observó una prevalencia de lactancia materna exclusiva de $3.4 \%$ en las últimas 48 horas en prematuros internados en la unidad neonatal, ${ }^{9}$ cifra muy baja comparada con países desarrollados, a pesar de ser una medida accesible de bajo costo. Por ello, es importante conocer en la perspectiva materna respecto a la lactancia en prematuros, sus ideas y sus creencias, que derivan de sus simbolizaciones y que ofrecen la posibilidad de explicar y, por lo tanto, comprender más a fondo sus elecciones de alimentación para el hijo prematuro y sus razones para modificarlas; todo ello con el propósito a futuro de diseñar o rediseñar, con base en la perspectiva de las madres, de mejora en los programas hospitalarios para incrementar el apego a la lactancia materna en nacidos prematuros. ${ }^{10}$

\section{OBJETIVO}

Comparar las representaciones maternas de salud y lactancia en hijos prematuros internados en hospitales de referencia, en tanto certificado o no como Hospital Amigo del Niño y de la Madre.

\section{MATERIAL Y MÉTODOS}

Estudio cualitativo efectuado en madres de niños prematuros hospitalizados para capturar las 
representaciones acerca de la lactancia materna en sus hijos, fundamentado en que las representaciones sociales generan opiniones, creencias y significados que se socializan y producen un impacto en las prácticas alimentarias. Además, son una forma de conocimiento socialmente elaborado y compartido, que se orienta hacia la práctica de la lactancia materna en prematuros. ${ }^{11,12}$

Lugar de estudio. Los hospitales de estudio son de referencia, atienden población del estado de San Luis Potosí y estados circunvecinos, tienen como misión la asistencia, enseñanza e investigación. El hospital de referencia con Iniciativa Hospital Amigo del Niño y de la Madre (IHANM) atiende, aproximadamente, 6,000 nacimientos al año y el hospital de referencia sin IHANM atiende cerca de 2,200 nacimientos al año. El índice de prematuridad es de alrededor de $10 \%$ en ambos. ${ }^{13}$

Contexto del estudio. Este artículo reporta los resultados de la primera fase del subproyecto: Intervención Vincular Educativa del Valor de Apego y Alimentación (VEVA) dirigido a las madres de recién nacidos prematuros hospitalizados en San Luis Potosí, derivada del macroproyecto multidisciplinario "Panorama epidemiológico, nuevos blancos y estrategias terapéuticas para enfermedades crónico-degenerativas asociadas con sobrepeso y obesidad en población pediátrica de San Luis Potosí". FMSLP-2012-C01-195024.

Muestra. La muestra fue intencional con base en el conocimiento de las unidades neonatales; del tipo de población que acude para la atención a prematuros; la madre porque en el ámbito de estudio es la que tiene permitido visitar a su hijo y el padre porque en estudios realizados ya aparece la figura paterna y su deseo de participar en el cuidado del hijo hospitalizado. Se buscó representatividad como madre adolescente, joven o adulta por la posible diferencia de ideas acerca del tópico en estudio. Se delimitó por saturación teórica, esto es, la recolección de datos finalizó cuando ya no aparecieron nuevos núcleos de sentido en las declaraciones de los entrevistados. El muestreo fue de voluntarios con consentimiento informado firmado, incluido un padre que manifestó su deseo de participar. ${ }^{14,15}$

Instrumentos. Se utilizó una guía de entrevista semiestructurada para recoger las comunicaciones de las madres y padres de prematuros hospitalizados. La primera parte contiene datos generales; la segunda parte, las preguntas detonadoras: ¿Qué es para usted la salud de su hijo prematuro internado? ¿Cómo alimentará a su hijo prematuro de acuerdo a su salud, lo que usted piensa y ha vivido en el hospital? El criterio de validez en investigación cualitativa se basó en que los cuestionamientos emergen del contexto de las diadas madre-padre-hijo prematuro hospitalizado. Las comunicaciones cumplieron el criterio de exhaustividad (saturación de datos), las categorías empíricas emergieron inductivamente de las comunicaciones de los propios participantes, denotando sus ideas y experiencias, en tal contexto que los posiciona como los informantes de mejor calidad en torno al fenómeno en estudio. ${ }^{15}$

Las entrevistas personales, cara a cara, se realizaron por personal de salud capacitado (curso-taller de 30 horas), con supervisión de la investigadora principal, en las salas de los hospitales facilitadas por las autoridades. Las entrevistas duraron aproximadamente 30 minutos, se audiograbaron y transcribieron íntegramente.

Procesamiento y análisis. En las comunicaciones se aplicó el análisis de contenido temático, transversalmente se aplicaron las categorías del referente (experiencias, creencias, informaciones e ideas de las madres sobre salud y alimentación del prematuro) para descubrir similitudes, divergencias y tendencias. El análisis se dividió 
en tres momentos: preanálisis (organización de la información, relectura de todo el texto que permitió impregnarse del contenido para aprehender las estructuras de relevancia); exploración del material para organizar los núcleos de sentido descubiertos en subtemas; $y$, tratamiento e interpretación o validación exhaustiva y crítica a la luz del referencial de interés. A partir de los subtemas se dio lugar a los temas. ${ }^{16}$ Se analizó el material de cada hospital y, posteriormente, se establecieron las comparaciones para descubrir similitudes o diferencias.

Consideraciones éticas y legales. Aprobaciones por Comités de Ética en Investigación de ambos hospitales: IHANM (HNM/2013-009); no IHANM (CEI-HGS-005-14), para cumplir los principios éticos y la Ley General de Salud en Materia de Investigación para la Salud. La población del estudio otorgó su consentimiento informado. Se aseguró la privacidad al usar siglas que identifican el tipo de hospital: Hospital Certificado (HC), Hospital No Certificado (HNC), palabra madre o padre, seguidas de la edad de los participantes al final de los testimonios. ${ }^{17}$

\section{RESULTADOS}

El conjunto de participantes voluntarios lo conformaron 31 informantes: 17 madres y un padre (hospital IHANM), y 13 madres (hospital sin IHANM). La edad promedio fue de 24.2 años $(\mathrm{DE} \pm 6.3)$; casadas-unión libre ( $85 \%)$; 6 con estudios de primaria (19\%); secundaria $19(59 \%)$; preparatoria $4(12.6 \%)$; profesional $1(3.1 \%)$ y 2 de otro tipo (un padre con posgrado) (6.3\%). Principalmente dedicadas a las labores del hogar. La media del peso al nacer de los prematuros fue 1701 g ( $\mathrm{DE} \pm 465$ g), tenían de 7 a 30 días de internamiento y algunos cerca de 2 meses.

Se develaron tres categorías o representaciones maternas: 1) la salud-corporalidad del hijo prematuro internado; 2) elección de la leche materna como mejor alimento versus fórmula para el hijo prematuro y 3) las informaciones recibidas acerca de la lactancia materna.

\section{La salud-corporalidad del hijo prematuro internado}

La salud del hijo prematuro en la visión de las madres-padre de ambos hospitales se fundamentó fuertemente en la corporalidad, tomando como referente a otros prematuros y a sus hijos anteriores. Los núcleos de sentido que componen esta representación en ambos hospitales coincidieron.

La corporalidad en función de tamaño, complexión y variaciones en el peso significó salud, como lo manifestaron las participantes de ambos hospitales:...Sana, muy bien, en comparación con los otros bebés que hemos visto sí está bien, está cachetona, chamorruda, tiene buen cuerpo... HC padre (26 años). Nada más estamos esperando que suba más para que ya me lo den de alta... HC madre (16 años). Comparado con mis anteriores bebés, pues está chiquito... ya lo veo más gordito, más cachetoncito... HNC madre (24 años). Asimismo, las variaciones en la coloración: al principio su coloración era amarilla y ya ahorita agarró un poco más de color, yo lo veo mejor... HC madre (19 años). Ya lo veo mejor, ya agarró más color, porque estaba pálido cuando estaba malito... HNC madre (24 años). Y la fuerza-vitalidad: yo lo veo bien, se está mueve y mueve a cada rato, eso quiere decir que sí tiene fuerzas... HC madre (34 años). Al principio muy decaído, sin fuercitas, sin nada, ahora es mucha la diferencia que como antes estaba... HC madre (20 años). En contraparte con la capacidad de supervivencia y al hijo imaginario, lo percibieron en fragilidad e indefensión: Ahorita ya está menos frágil que al principio porque hasta miedo me daba de tocarla de lo pequeñita que estaba y bien flaquita... HC madre (20 años). Sí llegué a pensar que no iba a vivir...lo veo indefenso... HNC madre (37 años). La veía demasiado frágil, 
me la imaginaba que naciera grande, que naciera fuerte... HNC madre (26 años).

Por su visión de salud, la afectividad materna se manifestó en sentimientos de desesperación y tristeza: ...En un principio sí me desesperaba, porque había pesado muy poquito; ya estando aquí bajó de peso, eso me angustió más, ya ha estado subiendo, pero ahorita otra vez que ya está succionando volvió a bajar porque es como si estuviera haciendo ejercicio... HC madre (19 años). Al inicio era muy triste, porque yo la veía muy pequeñita... HNC madre (26 años).

La falta de información para el cuidado del hijo generó temor dado que focalizaron la salud en el bienestar corporal:... HC [no fue mencionado]. Francamente me preocupa que tiene que tener cuidados especializados, el no estar yo informada, me va a dar angustia no saber... HNC madre (21 años).

\section{Elección del mejor alimento: leche materna versus fórmula para el hijo prematuro}

Para las madres y el padre en ambos hospitales la leche materna representó el mejor alimento para el prematuro y se manifestaron en favor de esta opción. En su visión concibieron la lactancia materna como la mejor opción por sus ventajas terapéuticas: la leche materna es lo mejor... es la mejor medicina... HC padre (26 años). No les da cólicos HC madre (26 años). Les ayuda mucho también a las defensas... HNC madre (37 años). Además, como fortificante que favorece el aumento de peso y desarrollo: les ayuda mucho al crecimiento y fortalecimiento HC madre (26 años). La mejor opción para los niños, para que crezcan más... HNC madre (20 años). Es lo único que puede ahorita subirlo de peso... HNC madre (32 años). Le confirieron el valor del vínculo de apego: la aceptan de cajón, ayuda mucho al apego entre la mamá y el hijo... HC madre (26 años).
Para su elección alimentaria compararon la leche materna con los sucedáneos, considerando la leche materna mejor que la fórmula: no hay problema en cuanto a fórmula alguna, simplemente sí es más benéfica mi leche materna... HC madre (21 años). En contraparte, se consideró mejor la fórmula de acuerdo al aumento de peso: ahorita me he acoplado muy bien con ella (fórmula), porque como trae muchas vitaminas para mi bebé entonces está subiendo muy bien de peso y no se me ha distendido ni nada, entonces pues ahí va con la fórmula... HC madre (30 años). En contrapunto, las madres les atribuyeron a los sucedáneos enfermedades: los bebés no las digieren bien... madre HC (16 años). A mi segundo bebé le daba fórmula 4 meses y me salió muy enfermizo y no subía de peso a comparación de la primera y de ella que están con puro pecho... HNC madre (24 años). A mi primer niño le di (fórmula) y sí me salió muy enfermizo, no me subía de peso... HNC madre (24 años). Las fórmulas lácteas pues tienen mucho químico no se la daría a mi hijo en ninguna situación... HC madre (21 años). (Fórmula) Dicen que les hace daño... HNC madre (20 años). (Fórmula) puede perjudicar a un bebé con el biberón... HNC madre (26 años). Se sopesó la accesibilidad económica de ambas opciones:... (leche materna) es accesible no tiene ningún costo... HC madre (26 años). (Fórmulas) ....son muy caras... HC madre (32 años). Es más gasto para uno... HNC madre (20 años).

Otro aspecto a decidir fue la duración de la lactancia materna: (la daría) como 4 meses... HC madre (19 años). Lo ideal como unos 2 años... HC madre (20 años). Le daría hasta el año... HC madre (34 años). Antes de que naciera mi bebé (planeaba) como mínimo darle 6 meses, pero ahorita como sé que nació prematura y por todas las complicaciones que tuvo, pues sí planeo darle el año, o hasta el año y medio si es posible... HNC madre (26 años). 
Las dificultades para mantener la lactancia materna y en las razones para suspenderla intervienen las situaciones hospitalarias prescriptivas: con mi leche más que nada, pero ahorita no me sale mucha, pero aparte no me han dejado que me lo pegue, para ver que síme saca... amamantaría si me sale y me dejan (personal médico) ... HC madre (34 años); y el poco contacto madrehijo que fomenta baja producción láctea:... pues con pura leche materna ahorita, al inicio sí le di pura leche materna, pero pues ya tengo 2 meses sin contacto completamente físico sin que me succione, entonces pues la disminución de mi producción es bastante, entonces ya es un poquito más complicado darle las 8 tomas al día de pura leche materna... HNC madre (26 años). La enfermedad materna podría conducir al abandono de la lactancia natural:... por enfermedad o por algo muy grave... HC madre (27 años). En una emergencia... HNC madre (24 años). Cuando el propio niño la rechace, como un indicador de que la leche materna ya no le es necesaria:... sería porque ya no necesita mucho (LM) cuando ya esté más grande y ya no la quiera... HC madre (19 años).

Optar por el cambio de leche materna a fórmula o combinarlas se justifica en las dificultades en la producción láctea: ... pues ahorita nomás le daría pecho y ya si en caso de que me diera cuenta de que él no se llena con mi leche, ya ahora sí le daría fórmula... HC madre (16 años). No tengo mucha producción de leche... HC madre (26 años). Porque a veces dice uno, porque no Ilena... HNC madre (24 años). Las actividades laborales maternas dificultarían mantener la lactancia: ...por el trabajo... HC padre (26 años). Que tuviera que trabajar, solamente por eso, si no, no se la quito... HNC madre (24 años). No continuaría tal vez por mis horarios en la escuela... HC madre (19 años). Continuaría usando la fórmula láctea, por el trabajo no puedo amamantarla todo el día... HC madre (26 años). Cuando se atribuye a la leche materna enfermedad en el hijo: ...no le cayó, como es laxante mi bebé empezó a hacer demasiada popó, ya traía hasta como sangrita en su popocita, entonces tuve que quitársela... HNC madre (30 años). Que le hiciera daño, que se enfermara... HNC madre (20 años). Le estaba dando leche materna, pero no le cayó bien, entonces ya se la cambié a fórmula... HNC madre (30 años). Si fuera necesario retirar el pecho por prescripción: a menos que se lo receten... HNC madre (20 años). Por el deseo materno de retirar la lactancia natural: ya para cuando uno les quiere quitar el pecho, les da uno la fórmula... HNC madre (24 años). Pues la leche materna, y un poco de lactancia, o sea de la leche normal de lata... HNC madre (21 años).

\section{Las informaciones recibidas acerca de la lactancia materna}

En ambos hospitales de estudio las madres-padre han recibido informaciones acerca de la lactancia materna. La importancia y beneficios de la lactancia natural son informaciones similares para las madres en ambos hospitales en donde se mencionó: ... que es la mejor alimentación... HC madre (19 años). Los beneficios nada más... HNC madre (37 años). Me han estado explicando los beneficios... HNC madre (26 años). La técnica de amamantar, fases y duración de la lactancia tienen lugar preponderante en las orientaciones que otorga el personal de salud: ... cómo agarrar el pecho y cómo sostener al bebé para que succione más... HC madre (24 años). Cómo se debe de amamantar... HNC madre (26 años). Para que baje la leche cómo hacer masajes... HNC madre (32 años). Más que nada me han dicho cómo darle... HNC madre (20 años). Todo lo que proporcionan los calostros, y las etapas que tiene pues sí también la leche materna, cuánto tiempo es lo recomendable... HNC madre (26 años).

La diferencia entre ambos hospitales se observa en lo pedagógico. Las didácticas de enseñanzaaprendizaje en el hospital certificado son 
diferentes desde comenzar tempranamente la enseñanza a la madre: ...se la pusieron (a amamantar), desde los primeros días... HC padre (26 años). La enseñanza en vivo ...fueron las enfermeras de clínica de lactancia a enseñarme como amamantar... HC madre (26 años). En el hospital certificado se cuenta con un proceso educativo sistematizado, evidente en el espacio definido dentro del hospital y programación de la acción educativa acerca de la lactancia materna así como la asignación de personal específico para realizarla: todos los miércoles ahí en el lactario nos hablan de lactancia materna... HC madre (20 años). Enseñan las enfermeras del lactario y clínica materna... HC madre (21 años). No se revela tal especificidad en el hospital no certificado: una plática sobre la lactancia... HNC madre (37 años). Soy primeriza y me he dado cuenta aquí en los informes que me dan, $y$ en los letreros que es muy saludable... HNC madre (21 años).

\section{DISCUSIÓN}

Para los participantes de los dos hospitales la salud del hijo prematuro estuvo representada en su corporalidad; la salud se asoció con el vigor físico, categoría de pensamiento colectivo aceptada socialmente; cualquier variación en sus características (peso, complexión, color, fuerza y movimiento) se tradujo en salud y supervivencia o enfermedad y fragilidad. ${ }^{12}$ El peso como criterio para el egreso fue construido en su vivencia de internamiento del prematuro, por tanto, si aumentaba, pudiera ser que más pronto "se los dieran"; así, las madres optaron por la lactancia materna.

La leche materna, representada como alimento "bueno", incluye la noción de sus bondades biológicas y psicológicas favorables sobre la salud-corporalidad y vínculo con el hijo prematuro, contenidos que se reconocen en los discursos del área de la salud. En contradicción, se representó la leche materna como alimento "malo" por trastornos como heces diarreicas o rastros de sangre. Así se desveló la creencia de que la leche materna puede ser dañina y "no caerle" al niño. Hallazgo similar fue reportado en un estudio con madres mexicanas egresadas de alojamiento conjunto de un instituto de alta especialidad, ${ }^{18}$ así como en madres libanesas. La leche es buena cuando el niño obtiene ganancia ponderal y no se queda con hambre y mala si perciben lo contrario, además del "veneno" que pueda tener. ${ }^{19}$ La percepción materna de producción insuficiente de leche frecuentemente es causa de abandono de lactancia, aunque la verdadera hipogalactia ocurre en menos de $10 \%$ de las mujeres. Estas percepciones modifican la decisión de otorgar lactancia materna hacia el uso de sucedáneos (aun pudiendo ser "alimento malo" [caro y que produce algunos trastornos]), así lo comunican al personal de salud, quienes en ese tenor pueden prescribir sucedáneos. ${ }^{20}$

Las madres atribuyeron el no amamantar también a la falta de contacto durante el internamiento del hijo por prescripción del personal; así, madres suecas percibieron como barrera al personal autoritario de la unidad neonatal. ${ }^{6}$

Las actividades maternas laborales podrían ser causa de abandono de la lactancia, así aconteció en $43 \%$ de las madres en un estudio efectuado en México sobre prevalencia de lactancia materna. ${ }^{2}$ Adicionalmente cambiarían su decisión de alimentar al seno materno en caso de enfermedad materna que requiriera medicamentos con efectos malos para el hijo, y también se ha reportado la enfermedad materna como una causa de destete precoz. ${ }^{20-22}$

El conocimiento materno sobre lactancia, así como las experiencias previas de amamantar, parecen no ser suficientes para lograr el apego a la misma, como se reportó en un estudio chileno y en un estudio en madres japonesas en el cual la experiencia previa de lactancia en multíparas no influyó en el mantenimiento de la misma. ${ }^{22,23}$ 


\section{CONCLUSIONES}

La salud del hijo prematuro se representa en la corporalidad, valorando mayormente al peso, si se incrementaba era indicio de salud y avance clínico satisfactorio o, por el contrario, si disminuía, era indicio de mayor fragilidad y posibilidad de fallecimiento, y para que incrementase el peso lo alimentarían con leche materna.

En las representaciones maternas se manifestó la construcción de conocimiento sobre la leche como alimento bueno, que les permitió optar inicialmente por alimentar al hijo con su leche. Coexiste la posibilidad de abandono por la idea de que la lactancia representa también dificultades como la percepción de producción insuficiente, la creencia de que la leche materna daña, la actividad laboral materna y la prescripción de sucedáneos.

El proceso de enseñanza-aprendizaje para fomentar la lactancia materna en cada hospital es diferente, mientras que en el HANM se denota sistematización en pro de la lactancia, con personal e infraestructura asignados a esa labor. En el hospital no certificado HANM se denotan solamente acciones informativas verbales o gráficas. Es deseable que el proceso de enseñanza-aprendizaje a las madres, padres y otros cuidadores, vaya más allá de la enseñanza tradicional, hacia enseñanza centrada en el adulto, incorporar talleres vivenciales conjuntando madre-padre, información impresa, en línea o de otro tipo que permita la consulta continua en el hogar sobre la lactancia.

Implementar la Iniciativa de Hospital Amigo del Niño y de la Madre con inclusión de la familia en un hospital amigable, la aplicación de la estrategia Save the Children con programa madre canguro y lactancia natural aplicada sistemáticamente, quizá incremente la prevalencia de lactancia materna exclusiva eficaz en los prematuros. Al mismo tiempo favorecería considerar los factores de abandono preventivamente para incentivar el apego a la lactancia natural exclusiva en los prematuros, así como el acompañamiento a las madres al menos los primeros seis meses postparto, dado que los factores de abandono pueden resolverse y así fortalecer la decisión de amamantar según la situación particular de cada diada.

Evaluar estas iniciativas sistemáticamente y aplicar estrategias de mejora con base en resultados de la evaluación e investigación, así como reforzar la sensibilización de los profesionales de la salud en la promoción de la lactancia materna exclusiva y reducción de la prescripción de sucedáneos, favorecería e incrementaría el apego a la lactancia en prematuros.

\section{LIMITANTES}

La representatividad por género no fue posible, pues solo un padre aceptó participar en el estudio. Es importante conocer la visión de los padres de prematuros en tanto que su función es indispensable en los vínculos entre progenitores y el establecimiento y continuidad de la lactancia materna. Es necesario ampliar la representatividad masculina y procurar estrategias de horarios accesibles a los padres para estudios futuros.

\section{AGRADECIMIENTOS}

Consejo Potosino de Ciencia y Tecnología (COPOCYT)-Proyecto VEVA FMSLP-2012-C01195424-Panorama epidemiológico, nuevos blancos y estrategias terapéuticas para enfermedades crónico-degenerativas asociadas a sobrepeso y obesidad de población pediátrica en San Luis Potosí.

\section{REFERENCIAS}

1. March of Dimes, PMNCH, Save the Children, WHO. Born Too Soon: The Global Action Report on Preterm Birth. Eds CP Howson, MV Kinney, JE Lawn. World Health Organiza- 
tion. Geneva, 2012. Acceso: http://www.who.int/pmnch/ media/news/2012/201204_borntoosoon-report.pdf.

2. Colchero MA, Contreras-Loya D, Lopez-Gatell H, Gonzáles $\mathrm{CT}$. The costs of inadequate breastfeeding of infants in México. Am J Clin Nutr. 2015;101:579-86. doi: 10.3945/ ajcn.114.092775.

3. Gutiérrez JP, Rivera-Dommarco J, Shamah-Levy T, Villalpando-Hernández S, Franco A, Cuevas-Nasu L, et al. Encuesta Nacional de Salud y Nutrición 2012. Resultados Nacionales. Cuernavaca, México: Instituto Nacional de Salud Pública (MX), 2012.

4. Quinlivan J, Kua S, Gibson R, McPhee A, Makrides MM. Can we identify women who initiate and then prematurely cease breastfeeding? An Australian multicentre cohort study. Int Breastfeed J. 2015;10:16. Doi: 10.1186/s13006015-0040-y.

5. Maastrup R, Moelholm Hansen Bo, Kronborg H, Norby Bojesen S, Karin Hallum, Annemi Frandsen, et al. Breastfeeding progression in preterm infants is influenced by factors in infants, mothers and clinical practice: the results of a National Cohort Study with High Breastfeeding Initiation Rates. PLOS ONE, 2014;9(9):e108208. https://doi. org/10.1371/journal.pone.0108208

6. Flacking R, Ewald U, Starrin B. "I Wanted to do a good job": Experiences of "becoming a mother" and breastfeeding in mothers of very preterm infants after discharge from a neonatal unit. Social Science \& Medicine, 2007;64:2405-2416.

7. Ericson J, Flacking R, Hellström-Westas L, Eriksson M. Changes in the prevalence of breastfeeding in preterm infants discharged from neonatal units: a register study over 10 years. BMJ Open. 2016;6(12):e012900. doi:10.1136/ bmjopen-2016-012900.

8. Barois J, Grognet S, Tourneux P, Leke A. Maternal and neonatal factors associated with successful brestfeeding in very preterm infants. Archives de Pédiatrie, 2013;20:969973. Doi: http://dx.doi.org/10.1016/j.arcped.2013.06.018.

9. Blanco-Cervantes P, Hoyos-Loya E, Álvarez-Hernández G, Ramírez-Rodríguez CA. Prevalencia de lactancia materna exclusiva en el servicio de neonatología de un Hospital Pediátrico de Sonora, México. Bol Clin Hosp Infant Edo. Son, 2015;32(1):3-9. http://www.medigraphic.com/pdfs/ bolclinhosinfson/bis-2015/bis151b.pdf.

10. Dosani $A$, et al. Breastfeeding the late preterm infant: experiences of mothers and perceptions of public health nurses. Int Breastfeed J. 2017;8(12):23. doi: 10.1186/ s13006-017-0114-0.

11. Gallegos-Martínez J, Reyes-Hernández J. Representations by caregivers, teachers, and children on food, nutrition, health, and school breakfast. Invest. Educ. Enferm. 2016;34(2):368-377. http://rccp.udea.edu.co/index.php/ iee/article/view/323272/20780816.
12. Andreatta MM. Social representations on the study of diet related to health. Diaeta, 2013;31(142): 42-49.URI: http:// hdl.handle.net/11336/4493.

13. Secretaría de Salud. Nacimientos ocurridos en hospitales de los Servicios de Salud del Estado de San Luis Potosí, 2011. Fuente: SINAC 2011. Subd. Informat. y Estadísticas en Salud.

14. Gallegos-Martínez J, Reyes-Hernández J, Silvan-Scochi CG. The hospitalized preterm newborn: meanings for parents of their participation in the Neonatal Unit. ISSN: 01041169 Rev Lat Am Enfermagem. 2013;21(6):1360-6. DOI: 10.1590/0104-1169.2970.2375.

15. Miguélez MM. Validez y confiabilidad en la metodología cualitativa. Paradigma, 2006;27(2):07-33.

16. Vaismoradi $\mathrm{M}$, Turunen $\mathrm{H}$, Bondas $\mathrm{T}$. Content analysis and the maticanalysis: Implications for conducting a qualitative descriptive study. Nurs Health Sci. 2013;15:398-405.

17. Secretaría de Salud. Nueva Ley General de Salud publicada en el Diario Oficial de la Federación el 7 de febrero de 1984 Texto Vigente. Última reforma publicada DOF. 2013:2501. http://www.ordenjuridico.gob.mx/Publicaciones/ CDs2006/CDMigracion/pdf/F1.pdf

18. Delgado-Becerra A, Arroyo-Cabrales LM, Díaz-García MA Quezada-Salazar CA. Prevalencia y causas de abandono de lactancia materna en el alojamiento conjunto de una institución de tercer nivel de atención. Bol Med Hosp Inf Mex, 2006;63:31-39. http://www.scielo.org.mx/scielo. php?script=sci_arttext\&pid=S1665-11462006000100005.

19. Osman H, El Zein L, WicK L. Cultural beliefs that may discourage breastfeeding among lebanese women: a qualitative analysis. Int Breastfeed J. 2009,4:12. Doi: 10.1186/17464358-4-12.

20. Oribe $M$, Lertxundi $A$, Basterrechea $M$, Begiristain $H$, Santa Marina L, et al. Prevalencia y factores asociados con la duración de la lactancia materna exclusiva durante los 6 primeros meses en la cohorte INMA Guipúzcoa. Gac. Sanit., 2015;29(1):4-9. http://dx.doi.org/10.1016/j. gaceta.2014.08.002.

21. Félix-Valenzuela JA, Martínez-Caldera E, Sotelo-Ham EI. Prevalencia y factores biosociales asociados al abandono de la lactancia materna exclusiva. Pediatría de México, 2011;13(2):47-56.

22. Pino SJL, López EMÁ, Medel IAP, Ortega SA. Factores que inciden en la duración de la lactancia materna exclusiva en una comunidad rural de Chile. Rev Chil Nutr. 2013;40(1):48-54. http://dx.doi.org/10.4067/S071775182013000100008.

23. Bai DL, Fong DYT, Tarrant M. Previous breastfeeding experience and duration of any and exclusive breastfeeding among multiparous mothers. Birth, 2015;42(1):70-77. Doi: 10.1111/birt.12152. 